\title{
Textsorten im Hörverstehenstraining für Deutsch als Fremdsprache
}

\author{
Thomas Stahl
}

The article offers considerations to an instruction in foreign language listening comprehension, which focuses the advancement of texttype competence as an element of listening comprehension competence. After basic remarks to the connection between communicative and texttype competence, the article deals with the relevance and function of texttypeorientation in listening comprehension. Finally the common practice in foreign language teaching concerning a texttype-oriented listening comprehension is regarded critically by a review of selected textbooks for German as a foreign language.

Listening comprehension - communicative competence - texttype - texttype competence

Hörverstehen - Kommunikative Kompetenz - Textsorten - Textsortenkompetenz

\section{Kommunikative Kompetenz und Textsortenkompetenz}

Sprache tritt immer in Gestalt von Texten auf, die man grundsätzlich bestimmten Textsorten zuordnen kann. Die jeweilige Textsorte mit ihrer spezifischen Intention bedingt Form und sprachliche Ausgestaltung des Textes. Sprache ist somit immer textsortenspezifisch (vgl. THURMAIR: 2001, 48). Demnach kann nur durch den Einsatz möglichst vieler unterschiedlicher Textsorten im Fremdsprachenunterricht gewährleistet werden, dass eine Vielfalt an sprachlichen Ausdrucksformen präsentiert wird, die dem natürlichen Spektrum zumindest nahe kommt und zu einem möglichst weit differenzierten sprachlichen Profil des Lerners beitragen kann. Dies ist in der Fremdsprachendidaktik weitgehend unumstritten. Dementsprechend präsentieren sich nahezu alle neueren DaF-Lehrwerke als spezifisch textsortenorientiert. In den Werbebroschüren der Verlage und in den Lehrerhandreichungen wird immer wieder auf die der Konzeption der Lehrwerke zugrunde liegende Textsortenorientierung hingewiesen. Zwar bieten die meisten Lehrwerke mittlerweile tatsächlich vielfältige Textsorten an, jedoch ist sicherlich THURMAIR $(2001,44)$ zuzustimmen, wenn sie schreibt: „Eine explizite oder auch nur implizite Arbeit an der Textsortenkompetenz hat in vielen Lehrwerken und damit auch an vielen Stellen des Deutsch-alsFremdspracheunterrichts keinen hohen Stellenwert."

Unter dem Begriff der Textsortenkompetenz ist die Fähigkeit zu verstehen, Textsortenwissen, also das Wissen über textsortenspezifische Elemente, in Kommunikationssituationen $\mathrm{zu}$ aktivieren und für kommunikative Ziele zu nutzen. Dabei ist zu bedenken, dass Textsorten „,konventionell geltende Muster für komplexe sprachliche Handlungen" (BRINKER: 2001, 135) darstellen, die sich zur Bewältigung verschiedenartiger kommunikativer Aufgaben mit je unterschiedlicher 
Intention entwickelt haben. Wenn die am Modell der kommunikativen Kompetenz orientierte Fremdsprachendidaktik als vorrangiges Lernziel die Befähigung zu angemessener Textproduktion und Textrezeption formuliert, dann kommt hierfür einer reflektierten Textsortenkompetenz eine zentrale Rolle zu. Denn stets haben wir es, wenn wir mit Texten produktiv oder rezeptiv umgehen, mit sortenbezogenem Wissen zu tun. Dieses unterstützt und vereinfacht als „Meta-Information“ oder „metakommunikative Hilfe“ (KALVERKÄMPER: 1983, 92) unser kommunikatives Handeln, das immer im Rahmen von Textsorten erfolgt. Denn Textsorten und das Wissen über sie geben den Kommunizierenden Orientierungshilfen für Prozesse des Textverstehens und der Textproduktion. Vereinfacht lässt sich sagen, dass Textsortenwissen die Kommunikation erleichtert (vgl. HUFEISEN: 2008, 51).

Dieses Textsortenwissen, das die Grundlage für Textsortenkompetenz darstellt, eignet man sich durch Texterfahrung im Laufe des Erwerbs der Muttersprache an. Ganz selbstverständlich wissen wir, ob wir es beim Radiohören mit einer Nachrichtensendung zu tun haben oder mit einem Werbespot. Wir können die Textsorten unterscheiden, ohne dass wir darin in theoretischer Hinsicht geschult wären. Im Hinblick auf Textsorten der Alltagskommunikation ist unser Textsortenwissen laut HUFEISEN $(2002$, 68) eher intuitiv und unreflektiert, wobei sicherlich ein Unterschied zwischen Textproduktion und Textrezeption zu erkennen ist. Gerade im Bereich der Rezeption kommt uns wahrscheinlich kaum zu Bewusstsein, wie sehr unsere Rezeptionshaltung durch „Textsortenmarker“ (HUFEISEN: 2008, 52) gesteuert wird.

Bislang wurde die Rolle und die Funktion der Textsortenkompetenz in der fachdidaktischen Literatur insbesondere für den Bereich der Schriftlichkeit mit dem Schwerpunkt Textproduktion thematisiert. Das Hörverstehen wurde in diesem Zusammenhang nur wenig in den Blick genommen. Textsortenwissen und damit Textsortenkompetenz beschränkt sich aber, wie HUFEISEN $(2008,51)$ ausführt, „keineswegs nur aufschriftlicheTexte, wir haben mindestens ebensoviele prototypische Muster von mündlichen Textsorten, von Gesprächen und Diskursen gespeichert.“ Und laut ADAMCZAK-KRYSZTOFOWITZ (2008, 43) ist ein potentieller Schwierigkeitsfaktor beim Hörverstehen „Vertrautheit bzw. fehlende Vertrautheit der Hörer mit der Textsorte“. Dazu kommt gerade in fremdsprachendidaktischen Zusammenhängen, dass viele Textsorten als konventionalisierte Rahmenvorgaben der Kommunikation kulturspezifisch ausgeprägt sind, sie also „von Kultur zu Kultur bzw. von einer Gesellschaft zur anderen unterschiedlich aussehen und verschiedene Formen sowie spezifische Textsortenmarker aufweisen“ (HUFEISEN: 2008, 52).

\section{Hörverstehen und die Rolle des Textsortenwissens}

Neben dem Leseverstehen, dem Sprechen und dem Schreiben ist das Hörverstehen eine der vier grundlegenden Kommunikationstätigkeiten. Der Begriff des Hörverstehens 
bezeichnet den durch einen auditiven Input ausgelösten Rezeptionsprozess, durch den der Inhalt mündlicher Texte erschlossen wird. Wie das Lesverstehen ist auch das Hörverstehen ein interaktiv-konstruktiver Prozess, bei dem Text(daten) und Hörer(wissen) interagieren. Einerseits gelangen datengeleitete Informationen vom Text zum Hörer. Dabei werden die phonologisch-prosodischen, lexikalischen und morphosyntaktischen Signale des akustischen Datenstroms registriert und aufbereitet. Andererseits nimmt der Hörer zum Text eine Erwartungshaltung ein: Er stellt Hypothesen über die inhaltliche und sprachliche Ausgestaltung nachfolgender Textteile an, überprüft, korrigiert und modifiziert diese im Verlauf des Hörprozesses. Dazu werden Schemata des Sprach- und Weltwissens aktiviert und an den Text herangetragen. Der Hörer kann so bestimmte Informationen antizipieren und Inferenzen bilden, also Fehlendes unter Nutzung der umgebenden Informationen und seines Wissens ergänzen. Datengeleitete und wissensgeleitete Operationen laufen im Hörverstehensprozess weitgehend parallel ab und ihr Zusammenspiel führt letztlich zum Textverständnis.

Ein kompetenter Hörer kann datengeleitete und wissensgeleitete Prozesse in optimaler Weise zusammenfügen, vor allem kann er sein Wissen und seine Kenntnisse zur gezielten und damit rationell-effektiven Decodierung und Verarbeitung nutzen. Die Bedeutung des erwartungs- bzw. wissensgeleiteten Verarbeitungsprozesses beim Hörverstehen in der Fremdsprache wird evident, wenn man sich die Störung der datengeleiteten Informationsverarbeitung aufgrund fehlenden bzw. zu wenig ausgeprägten Sprachwissens vor Augen führt. Als bedeutender Faktor des wissensgeleiteten Rezeptionsprozesses wirkt dabei die Textsortenkompetenz des Hörers. Denn die Fähigkeit, einzelne Textsorten zu erkennen und einzuordnen, führt zu einer festen Rezeptionshaltung, was wiederum die Hypothesenbildung fördert und das Antizipieren sowie das Inferieren unterstützt. Die Zuordnung eines Textes zu einer Textsorte aktiviert beim Hören bestimmte Erfahrungsraster im Hinblick auf Funktion und Form. Diese entlasten den Rezeptionsprozess, indem der Focus auf inhaltliche Aspekte gerichtet werden kann (vgl. HUFEISEN: 2008, 51).

TextsortenmerkmalehelfendemHörerdarüberhinaus, Kommunikationsabsichten zu erkennen und beeinflussen maßgeblich die Wahl eines adäquaten Hörstils. Denn es ist neben der Hörintention des Hörers die Textsorte, die die Art und Weise, wie man einen konkreten Text rezipiert, determiniert. In authentischen Hörsituationen werden Texte je nach Textsorte unterschiedlich gehört. Man hört einen Fachvortrag sicherlich anders als einen Werbespot, diesen wiederum anders als eine Durchsage auf dem Bahnhof. In der Fachliteratur werden drei grundsätzliche Hörstile unterschieden, wobei die Begriffe nicht einheitlich verwendet werden: Das globale oder kursorische Hörverstehen bezieht sich auf das generelle Erfassen der Struktur, der Gesamtaussage oder der Schlüsselbegriffe eines Hörtextes. Um selektives oder selegierendes Hörverstehen handelt es sich, wenn das Ziel das Erfassen bestimmter 
Einzelinformationen eines Hörtextes ist $^{1}$. Beim detaillierten oder intensiven Hörverstehen wird versucht, einen Hörtext im Detail zu erfassen, um ein möglichst vollständiges Textverständnis zu erreichen. ${ }^{2}$

Ob ich nun einen Text global höre, ob ich beim Hören selektiv oder orientierend vorgehe, ist abhängig von meiner Leseabsicht, die wiederum in unauflösbarem Zusammenhang mit der jeweiligen Textsorte steht. Beispielsweise wird man sicherlich als Reisender, der sich für die Abfahrtszeit eines bestimmten Zuges interessiert, eine Bahnhofsdurchsage kaum detailliert hören, sondern eher einen selektiven Hörstil nutzen. Ein kompetenter Hörer ist in der Lage, seinen Hörstil auf die jeweilige Textsorte abzustimmen und flexibel im Laufe des Rezeptionsprozesses anzupassen. Folgerichtig gilt der textsortenadäquate Einsatz der Hörstile als bedeutende Hörverstehensstrategie, deren Förderung ein wichtiger Faktor für die Entwicklung der Hörverstehenskompetenz in der Fremdsprache darstellt. Die Vermittlung der verschiedenen Hörstile gehört konsequenterweise zu den wichtigen Lernzielen der kommunikativen Fremdsprachendidaktik.

\section{Textsortenorientierte Hörverstehensübungen im Unterricht Deutsch als Fremdsprache}

Wenn das Lernziel des Fremdsprachenunterrichts kommunikative Kompetenz heißt, dann müssen Lernende auch im Bereich des Hörverstehens mit einer Vielzahl der in der Realität vorkommenden Textsorten konfrontiert werden. Hörverstehensübungen dürfen sich deshalb bereits im Grundstufenbereich nicht nur auf das Hören von dialogischer Alltagskommunikation beschränken, sondern müssen, nimmt man den Begriff der kommunikativen Kompetenz ernst, unterschiedlichen Textsorten sowohl der direkten Kommunikation als auch der indirekten Kommunikation gewidmet sein. $\mathrm{Zu}$ denken ist dabei neben den klassischen Dialogen beispielsweise an Telefonansagen, Durchsagen, Interviews, Nachrichten, Berichte, Hörspiele etc. Gehört werden muss natürlich auch bei der Rezeption audiovisueller Medienangebote wie Film und Fernsehen, wodurch das Zusammenspiel von Hören und Sehen und die Fertigkeit des so genannten Hörsehverstehens in den Blick geraten.

Um die Textsortenkompetenz der Lernenden zu entwickeln, ist es nötig, Übungen zum Hörverstehen textsortenorientiert zu gestalten. WIEMER (1999, 42) plädiert in ihrer Darstellung zur Didaktik des fremdsprachlichen Hörverstehens dafür, Übungen anzubieten, die „nicht nur auf den komplexen Hörprozess

1 EGGERS (1996, 20) differenziert zwischen dem durch gezielte Vorgaben gelenkten selektiven Hörverstehen und dem allein durch die Verstehensabsichten des Rezipienten gesteuerten selegierenden Hörverstehen.

2 Andere in der Fachliteratur genannte Hörstile sind das orientierende (SOLMECKE: 1993, 25), das reflektierende (EGGERS: 1996, 22), das totale (EGGERS: 1996, 21) und das analytische Hörverstehen (WENDT: 1988, 17). 
ausgerichtet sind, sondern deren Ziel es ist, einzelne, konstitutive Teile des komplexen Hörverstehensprozesses zu trainieren." Dazu gehöre auch, so WIEMER (1999, 43), das „Erkennen von Textsorten“. Ein unterrichtspraktisches Beispiel hierfür findet sich im Lehrwerk Optimal A1 (MÜLLER et al.: 2004, 41). Die Lernenden sollen aufgrund von Abbildungen Erwartungen an drei Hörtexte - Sonderangebote im Supermarkt, Börsenkurse, Radiokochkurs - formulieren. Dadurch werden zugehörige Textsortenschemata aktiviert, und den Lernenden wird klar, welche Art von Informationen sie in welcher Form hören werden. Die Arbeitsanweisung lautet dann: „Hören Sie. Notieren Sie: Preise, Zahlen, Gewicht, ... “ Das aktivierte allgemeine Muster der Textsorte wird durch den jeweiligen Hörtext mit konkreten Daten gefüllt, deren Rezeption durch eine feste und der Textsorte angemessene Rezeptionshaltung erleichtert wird.

Die explizite Arbeit an der Textsortenkompetenz zielt ab auf den Aufbau eines metakognitiven Bewusstseins. Um dies zu erreichen, müssen die in Hörübungen eingesetzten Hörtexte über eine textsortentypische Makrostruktur verfügen und typische Textsortenmarker (vgl. HUFEISEN: 2008, 51) aufweisen. Darüber hinaus ist es im Hinblick auf Entwicklung und Förderung zielsprachlicher Hörverstehenskompetenz notwendig, dass im Fremdsprachenunterricht Hörtexte unter natürlichen Rezeptionsbedingungen der jeweiligen Textsorte gehört werden und ihre Bearbeitung stärker an authentischen, das heißt textsortentypischen Verwendungssituationen, orientiert sein muss. ${ }^{1}$ Hörverstehensaufgaben müssen authentische oder eine an der Realität orientierte Simulation von Höraktivitäten ermöglichen. Dazu müssen die Hörtexte selbst funktional sein und mit funktionalen und der Textsorte angemessenen Hörübungen versehen werden, wenn sie sinnvoll eingesetzt werden sollen. Die folgende Aufgabe aus dem Lehrwerk eurolingua Deutsch 2. Neue Ausgabe (EISOLD et al.: 2006, 36) simuliert eine solche, quasi-authentische' Hörsituation zumindest in Teilen: „Durchsagen am Bahnhof. Sie wollen mit dem InterCityExpress von Kassel nach München fahren. Auf welche Wörter müssen Sie achten? Kreuzen Sie dann an: Welche Durchsagen sind wichtig für Ihre Fahrt?" Da Durchsagen auf dem Bahnhof sicherlich mehrmals durchgegeben werden, lautet konsequenterweise der zweite Teil dieser Höraufgabe folgendermaßen: „Hören Sie die Durchsagen noch zweimal. Notieren Sie die Informationen zu Ihrem Zug nach München [...]." Die Fortführung der Aufgabenstellung mit der Anweisung, möglichst viele Informationen zu den anderen Durchsagen zu notieren, entspricht dann jedoch nicht mehr authentischen Rezeptionsbedingungen, sondern ist lediglich unterrichtlichen Aspekten geschuldet. Aber Höraufgaben, so ist auch für SOLMECKE $(2003,10)$ klar, sollten stets „Hörverstehensvorgänge anregen, die dem jeweiligen Text auch in außerunterrichtlichen Situationen angemessen sind“ und z. B. keine unwichtigen Details abfragen.

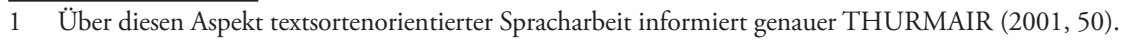


Ein entscheidender Vorteil eines an authentischen Bedingungen orientierten Hörverstehenstrainings ist es, dass sich unterschiedliche Hörstile bewusstmachen und textsortenbezogen trainieren lassen. Dadurch lässt sich zugleich der insbesondere im Grundstufenbereich häufig anzutreffenden Tendenz zum ,Wort-für-WortHören-und-Verstehen-Wollen' entgegenwirken. In authentischen Hörsituationen (in der Muttersprache) hört man Texte Wort für Wort eigentlich nur dann, wenn man ihn auswendig lernen möchte. Fremdsprachenlernende „kleben“ jedoch oft ,an der Textoberfläche und bemühen sich, Wort für Wort und möglichst alle Informationen zu behalten“ (BRAUN: 2008, 72). Diese Rezeptionshaltung ist, hält man sich dazu die Flüchtigkeit des auditiven Inputs vor Augen, oftmals ein entscheidender Grund für das Ausbleiben von Hörverständnis. Denn der Versuch, sämtliche Textdaten zu dekodieren, führt meist zu einem Bruch im Verstehen, wenn der Text unbekanntes Sprachmaterial enthält und beim Lerner Prozesse der Worterkennung oder der syntaktischen und semantischen Analyse von Wortfolgen wenig routinisiert sind. Das Arbeitsgedächtnis wird bei diesem Versuch derart überfrachtet, dass das aufgenommene und dekodierte Sprachmaterial zerfällt, bevor es mit den vorhandenen Wissensstrukturen verbunden werden kann.

Durch den Unterricht sollen die Lernenden in die Lage versetzt werden, für sie neue Texte selbstständig zu erschließen, auch dann, wenn diese Texte unbekanntes Sprachmaterial beinhalten. Dazu benötigen sie Strategien, mit deren Hilfe geringe Sprachkenntnisse kompensiert werden können. Wichtig ist, dass es im Unterricht nicht nur um die Anwendung und das Einüben von Rezeptionsstrategien gehen kann, sondern auch die Bedeutung solcher Strategien bewusstgemacht werden muss, sodass sie von den Lernenden mehr und mehr selbstständig im eigenen Hörprozess eingesetzt werden können. Es geht also um den Aufbau von strategischer Kompetenz. Dafür ist es notwendig, verstärkt das Augenmerk auf den Hörprozess zu richten. Nicht das Produkt, also die Verstehensleistung am Ende des Hörvorgangs, sondern der Weg des Verstehens steht in einem strategieorientierten Unterricht im Mittelpunkt. So formuliert auch MARX (2005, 152): „Allgemeines Strategienwissen und -können verhilft zu einem verbesserten Spracherwerb überhaupt [...], aber bewusst vermittelte, für das Hörverstehen speziell geeignete Strategien können Wesentliches zum Erfolg eines fremdsprachlichen Hörers beitragen."

Der Einsatz des Textsortenwissens und die Nutzung der Textsortenkompetenz beim Hörverstehen ist sicherlich eine zentrale Hörverstehensstrategie. Textsortenorientierte Hörverstehensübungen bieten Raum für eine explizite Arbeit an der Textsortenkompetenz und die Bewusstmachung der Bedeutung des Textsortenwissens für den Rezeptionsprozess.

Bei einem Blick in aktuelle Grundstufenlehrwerke auf dem Niveau A1 und A2 fällt auf, dass die meist auch hier postulierte Textsortenvielfalt im Bereich des Hörverstehens erstaunlich dünn ausfällt. Eindeutig dominieren hier Hörverstehensaufgaben des Modells „Hören Sie das Gespräch“. Formen der 
monologischen bzw. indirekten Kommunikation finden sich nur sporadisch. So finden sich in dem sieben Lektionen umfassenden ersten Band des Lehrwerks Schritte (BOVERMANN et al.: 2003, 46) neben dialogischen Hörtexten lediglich in zwei Höraufgaben der Lektion 5 die Textsorte Infospot bzw. Ansage (auf Anrufbeantworter). Auch findet, im Gegensatz zum Bereich des Lesens, in vielen Lehrwerken auf diesem Niveau kein strategieorientiertes Hörverstehenstraining, in dem Verstehensstrategien vor/während/nach dem Hören vermittelt werden und auch die Arbeit an der Textsortenkompetenz der Lernenden eine Rolle spielt, nicht statt.

Diese Feststellung gilt in ganz besonderem Maße für das 2001 erschienene Lehrwerk Delfin, das an dieser Stelle beispielhaft herangezogen werden soll. Im Vorwort (AUFDERSTRASSE et al.: 2001,3) versprechen die Autoren dann auch nur für den Bereich des Leseverstehens Textsortenvielfalt: „Hier finden Sie attraktive Lesetexte verschiedenster Textsorten. Dazu Übungen, die Ihnen beim Auffinden und Verstehen der wichtigen Inhalte helfen. "1 Hörverstehen dagegen könne, so das Vorwort, gezielt in „alltagsnahe[n] Gesprächssituationen“ trainiert werden. Und tatsächlich werden die angebotenen dialogischen Texte, die zwar gelegentlich auch am Telefon stattfinden und ab Lektion 7 hin und wieder als Interview daherkommen, nur sporadisch ergänzt durch eine Nachrichtensendung in Lektion 12 (AUFDERSTRASSE et al.: 2001, 122) und einen Wetterbericht in Lektion 13 (AUFDERSTRASSE et al.: 2001, 132). ${ }^{2}$ Schon aufgrund dieser Beobachtung kann festgestellt werden, dass eine Arbeit an der Textsortenkompetenz in diesem Lehrwerk nicht ausdrücklich intendiert ist. Dazu kommt noch, dass die begleitenden Übungen, die dem Hörverstehen gelten, meist nur das Verständnis überprüfen, also rein produktorientiert und - ohne immensen Aufwand des/der Unterrichtenden kaum dazu geeignet sind, Hörverstehensstrategien aufzubauen, geschweige denn bewusstzumachen. Der Hörprozess selbst wird in Delfin an keiner Stelle zum Lerngegenstand. Fast ausschließlich werden Aufgaben nach dem Hören angeboten, die das Verständnis mehr überprüfen als entwickeln. Aktivitäten vor und nach dem Hören finden sich nicht und explizit thematisiert werden Verstehensstrategien an keiner Stelle. Bewusstmachende und konfrontative Textsortenarbeit ist nicht vorgesehen. Den angebotenen „alltagsnahe[n] Gesprächssituationen“ ist darüber hinaus kaum Authentizität zuzuschreiben, dafür bleibt die in den Gesprächen verwendete Sprache zu starr an bis dahin vermittelten Wortschatz- und Grammatikeinheiten orientiert und wirkt zu synthetisch. Beim näheren Hinsehen

1 In ihrer Beurteilung bescheinigen COOLS/SERCU $(2006,11)$ dem Lehrwerk Delfin jedoch insgesamt eine gering ausgeprägte Textsortenvarianz. Der Schwerpunkt des Lehrwerks liege eher auf der Grammatik- und Wortschatzvermittlung und die Progression sei „in Bezug auf Textsorten, Übungstypen und Authentizität eher beschränkt.“

2 In den Lektionen des Lehrwerks, die dem Niveau B1 zuzuordnen sind (ab Lektion 14) erhöht sich die Zahl unterschiedlicher Textsorten geringfügig. 
erkennt man auch, dass Hörtexte und Höraufgaben keineswegs immer nur dem Hörverstehen dienen. Vielfach wird damit ein bestimmtes grammatisches Phänomen transportiert und eingeübt. Derartige "Lerntexte“ eignen sich jedoch kaum als Hörverstehenstexte, wie SOLMECKE $(2003,8)$ folgendermaßen begründet: „Das Textverstehen ist hier nicht Zweck, sondern Zwischenstadium, und da der Text Modellcharakter haben soll, ist es zwangsläufig ein wortwörtliches Hören und Verstehen, bei dem die Aufmerksamkeit weniger auf den Inhalt als auf die Sprache gerichtet ist. So notwendig dieser Umgang mit Texten einerseits ist, verstärkt er andererseits die [...] Tendenz, jeden Text wortwörtlich verstehen zu wollen und bei jedem unverstandenen Wort, jeder grammatischen Schwierigkeit stecken zu bleiben. Auf diese Weise werden Gewohnheiten geschaffen, die dem sinnentnehmenden Hören erheblich im Wege stehen."

Die sich in Delfin offenbarenden Tendenzen zeigen sich auch in anderen Grundstufenlehrwerken für Deutsch als Fremdsprache, wenn auch meist nicht so deutlich. Strategieorientiertes Lernen, das für den Bereich des Lesens meist ermöglicht wird, ist für das Hörverstehen nur selten intendiert. Bei vielen Hörverstehensaufgaben ist darüber hinaus eine authentische und textsortenspezifische Rezeptionshaltung nicht erkennbar. Eindrucksvoll beweist dies die Aufgabe „Frau Egli, Herr Egli, die Kellnerin: eine Bestellung" aus dem ersten Band des Lehrwerks Passwort Deutsch (ALBRECHT et al.: 2001, 50), die sicherlich ein Extrembeispiel einer verfehlten Hörverstehensaufgabe darstellt. Die Arbeitsanweisung lautet: „Wer spricht? Bitte hören Sie und schreiben Sie die Namen." Die Lerner sollen also entscheiden, wer

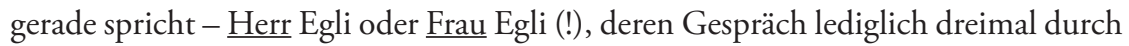
die Kellnerin unterbrochen wird. Die Felder vor den jeweiligen Gesprächsteilen, in die die Lerner die Namen eintragen sollen, sind zu allem Überfluss für die drei Personen unterschiedlich gestaltet (Länge, Farbe), so dass Hörverstehen im eigentlichen Sinn zum Lösen dieser Aufgabe wohl überhaupt nicht notwendig ist. Abgesehen von der Qualität des Hörtextes selbst (ein rein synthetischer Lehrbuchtext) ist jedenfalls nicht erkennbar, welche Hörverstehensleistung in dieser Aufgabe angesprochen wird und auf welche außerunterrichtliche Hörsituation eine so konzipierte Aufgabe vorbereiten soll.

\section{Literatur:}

ADAMCZAK-KRYSZTOFOWICZ (2008): Adamczak-Krysztofowicz, Sylwia. Hörverstehenskompetenz erwachsenengemäß entwickeln. Konsequenzen aus einer qualitativen Kursteilnehmerbefragung für die Hörtextauswahl im DaF-Unterricht, in: Zielsprache Deutsch, 2008, 35, 1, 36-59.

ALBRECHT u.a. (2001): Passwort Deutsch. Kurs- und Übungsbuch. Stuttgart: Ernst Klett International, 2001.

AUFDERSTRASSE u.a. (2001): Aufderstraße, Hartmut u.a. Delfin. Lehrwerk für Deutsch als Fremdsprache. Lehrbuch, Ismaning: Hueber, 2001. 
BOVERMANN et al. (2003): Bovermann, Monika et al. Schritte 1. Deutsch als Fremdsprache. Kursbuch + Arbeitsbuch. Ismaning: Hueber, 2003.

BRINKER (2001): Brinker, Klaus. Linguistische Textanalyse. Eine Einführung in Grundbegriffe und Methoden. Berlin: Erich Schmidt Verlag, 5. Auflage, 2001.

COOLS/SERCU (2006): Cools, Dorien/Sercu, Lies. Die Beurteilung von Lehrwerken an Hand des Gemeinsamen Europäischen Referenzrahmens für Sprachen: Eine empirische Untersuchung von zwei kürzlich erschienenen Lehrwerken für Deutsch als Fremdsprache, in: Zeitschrift für Interkulturellen Fremdsprachenunterricht, 2006, 11, 3, 17 S. [abrufbar unter http://www.ualberta.ca/_german/ejournal/Cools_Sercu1.htm; Stand 31.3.2009]

EGGERS (1996): Eggers, Dietrich. Hörverstehen: Bestandsaufnahme und Perspektiven, in: Kühn, Peter (Hg.): Hörverstehen im Unterricht Deutsch als Fremdsprache. Theoretische Fundierung und unterrichtliche Praxis. Frankfurt/M.: Lang, 13-44.

EISOLD et al. (2006): Eisold, Knut et al. eurolingua Deutsch 2. Neue Ausgabe. Teilband 1. Berlin: Cornelsen Verlag, 2006. 